\title{
Heat diffusion across a local stochastic magnetic field
}

\author{
Q. Yu \\ Max-Planck-Institut für Plasmaphysik, EURATOM Association, D-85748 Garching, \\ Germany
}

\begin{abstract}
Based on the earlier work for a single magnetic island and a weak stochastic magnetic field (Yu 2006 Phys. Plasmas 13 062310), the heat diffusion across a local fully stochastic magnetic field is studied numerically and compared with the analytical theories. The ratio between the parallel and the perpendicular heat diffusivity, $\chi_{\|} / \chi_{\perp}$, is found to be an important parameter in characterizing the transport. With the increase in $\chi_{\|} / \chi_{\perp}$, the transport is dominated first by the additive effect of individual islands and then by the field ergodicity and $\chi_{\|}$.
\end{abstract}

PACS numbers: 52.25.Fi, 52.65.Kj, 52.55.Dy, 52.35Vd

\section{Introduction}

Magnetic islands generally exist in tokamak plasmas, caused either by the tearing mode type instabilities or by the externally applied resonant helical field [1-9]. When there are magnetic perturbations of different helicities and their corresponding rational surfaces are sufficiently close, the local magnetic field becomes stochastic. In this case strong interaction between these perturbations and the subsequent change in the plasma energy confinement have been observed $[3,4]$. In addition, the transport across the stochastic magnetic field boundary in stellarators and tokamaks is also an important topic and has attracted extensive studies [6-9], and the field stochasticity could even lead to anomalous radial heat transport [10-16]. Therefore, the heat diffusion across the stochastic field is of general interest in plasma physics.

The degree of the field stochasticity is usually described by the parameter

$$
\Delta=\left(W_{1}+W_{2}\right) /\left(2\left|r_{1}-r_{2}\right|\right),
$$

where $W_{1}$ and $W_{2}$ are the widths of two neighbouring islands and $r_{1}$ and $r_{2}$ are the minor radius of the corresponding rational surfaces. The field stochasticity starts approximately from $\Delta=1[10,11]$. Assuming the original electron temperature in the absence of the stochastic field, $T_{\mathrm{e} 0}$, has a radial gradient being perpendicular to the original magnetic surface, the effective radial heat diffusivity in a fully stochastic field, $\chi_{\mathrm{e}}$, can be defined by the relation

$$
q_{r}=-\chi_{\perp} T_{\mathrm{e} 0}^{\prime}=-\chi_{\mathrm{e}} T_{0 / 0}^{\prime}
$$

due to the conservation of the radial heat flux $q_{r}$, where $\chi_{\perp}$ is the perpendicular heat diffusivity, the prime is for $\mathrm{d} / \mathrm{d} r$ and $T_{0 / 0}^{\prime}$ is the averaged (along the poloidal and the toroidal directions) electron temperature gradient in the stochastic field. The enhanced radial heat conductivity due to the magnetic field ergodicity is then given by

$$
\chi_{r}=\chi_{\mathrm{e}}-\chi_{\perp}=\chi_{\perp}\left(T_{\mathrm{e} 0}^{\prime} / T_{0 / 0}^{\prime}-1\right) .
$$

It was shown by Rechester and Rosenbluth that [10] $\chi_{r}$ equals $D_{\mathrm{M}} v_{\mathrm{Te}}$ in the collisionless regime (the electron mean free path $L_{\lambda}$ is longer than the Kolmogorov length $\left.L_{k} \approx\left[L_{\mathrm{s}}^{2} /\left(k_{\perp}^{2} D_{\mathrm{M}}\right)\right]^{1 / 3}[10-16]\right)$, where $v_{\mathrm{Te}}$ is the electron thermal velocity, $k_{\perp}$ is the perpendicular wave vector of the perturbations, $L_{\mathrm{s}}=R q^{2} / r q^{\prime}, q$ is the safety factor, $D_{\mathrm{M}}=$ $L_{0} \sum\left(b_{r, k} / B_{0 \mathrm{t}}\right)^{2} \delta\left(m_{k} / q_{k}-n_{k}\right), b_{r, k}, m_{k}$ and $n_{k}$ are the radial magnetic field perturbation, the poloidal and toroidal mode numbers of the kth Fourier component, respectively, $B_{0 \mathrm{t}}$ is the toroidal magnetic field, $L_{0} \approx \pi R$ and the summation is over $k$ to include contributions from all resonant components. While in the collisional regime $L_{\lambda}<L_{\kappa}$,

$$
\chi_{r}=D_{\mathrm{M}} \chi_{\|} / L_{c \delta},
$$

where $L_{c \delta}=L_{c} \ln \left[\left(r / m L_{c}\right)\left(\chi_{\|} / \chi_{\perp}\right)^{1 / 2}\right]$ and $L_{c}=$ $\pi R / \ln (\pi \Delta / 2)[10]$.

Krommes et al later showed that the collisional regime consists of three sub-regimes [11]. With the decrease of $L_{\lambda}$ they are

(a) the Rechester-Rosenbluth regime (valid for $\tau_{\|}<\tau_{k}<$ $\left.\tau_{\perp}\right)$ :

$$
\chi_{r}=D_{\mathrm{M}} \chi_{\|} / L_{k}
$$

where $\tau_{\|}=L_{0}^{2} / \chi_{\|}, \tau_{k}=L_{k}^{2} / \chi_{\|}$and $\tau_{\perp}=1 /\left(k_{\perp}^{2} \chi_{\perp}\right)$,

(b) the Kadomsev-Pogutse regime (valid for $\tau_{\|}<\tau_{\perp}<\tau_{k}$ ):

$$
\chi_{r}=D_{\mathrm{M}}\left(\chi_{\|} \chi_{\perp}\right)^{1 / 2} / k_{\perp} \text { and }
$$




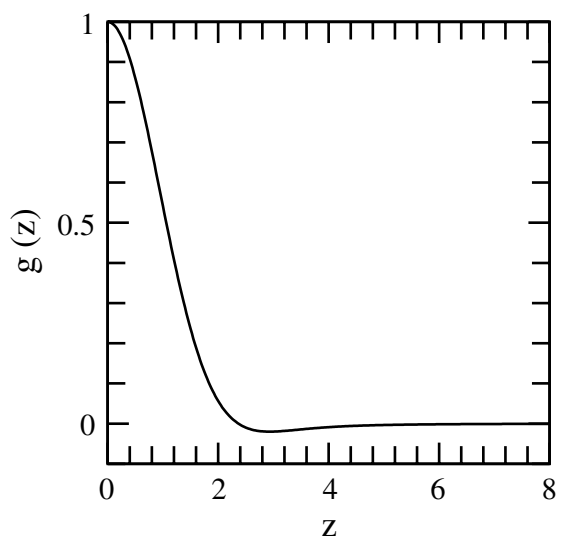

Figure 1. $g(z)$ versus $z \cdot g(z)=1$ for $z=0$ and approaches zero for $z>2$.

(c) the fluid regime (valid for $\tau_{\perp}<\tau_{\|}<\tau_{k}$ ):

$$
\chi_{r}=D_{\mathrm{M}} \chi_{\|} / L_{0}
$$

Equations (4)-(7) are the conventional formulae for $\chi_{r}$ in the collisional regime $[15,16]$. For obtaining these results, various assumptions have been made [10-13], leading to the difference between these results, such as the difference between equations (4) and (5) [10-16].

It was found recently that [17] in the limit $\chi_{\|} / \chi_{\perp} \gg 1$ but $W_{k} / W_{c, k} \ll 1$,

$$
\chi_{r}=\sum_{k} \chi_{\perp} \frac{W_{k}^{4}}{8 W_{c, k}^{4}} g_{k}\left(z_{k}\right)=\sum_{k} \chi_{\|} \frac{b_{r, k}^{2}}{2 B_{0 \mathrm{t}}^{2}} g_{k}\left(z_{k}\right),
$$

where

$$
\left.W_{k}=4\left[b_{r, k} L_{q} R / B_{0 \mathrm{t}} n_{k}\right)\right]^{1 / 2}
$$

being the island width due to the $k$ th component magnetic perturbation, $L_{q}=q / q^{\prime}$,

$$
W_{c, k}=a\left(\chi_{\perp} / \chi_{\|}\right)^{1 / 4}\left[8 L_{q} /\left(\varepsilon a n_{k}\right)\right]^{1 / 2}
$$

being the heat diffusive layer width at the $k$ th rational surface, $\varepsilon=a / R$,

$$
z_{k}=2^{3 / 2}\left(r-r_{s, k}\right) / W_{c, k}
$$

being the normalized (to $W_{c, k} / 2^{3 / 2}$ ) distance from the $k$ th rational surface $r_{s, k} . g(z)=[1+z f(z)]$ and $f(z)=$ $-0.5 z \int \mathrm{d} y\left(1-y^{2}\right)^{-1 / 4} \exp \left(-z^{2} y / 2\right)$ with the integration from 0 to 1 [17]. The function $g(z)$ is shown in figure 1 . to [17]

For a single island, equation (8) is also valid and reduces

$$
\chi_{r}=\chi_{\perp} \frac{W^{4}}{8 W_{c}^{4}} g(z)=\chi_{\|} \frac{b_{r}^{2}}{2 B_{0 \mathrm{t}}^{2}} g(z) .
$$

Equations (8) and (12) indicate that $\chi_{r}$ is dominated by the additive effects of these individual islands when $W_{k} / W_{c, k} \ll 1$.

Differing from equations (4)-(7), the $\chi_{r}$ given by equation (8) is a local enhanced radial heat conductivity due to magnetic field ergodicity and is dominated by the additive effects of these individual islands satisfying $z_{k}<2$, since $g_{k}\left(z_{k}\right)$ varies along the minor radius and approaches zero for $z_{k}>2$ as shown in figure 1. While the $\chi_{r}$ given by equations (4)-(7) includes contributions from all resonant perturbations. Only in the limit of a sufficiently small $z_{k}$ such that $g_{k}\left(z_{k}\right) \approx 1$, equations $(8)$ reduces to the fluid regime result, equation (7), except for a factor of $1 / 2$.

The valid regime of equation (8) is even more different from those of equations (5)-(7). To be self-consistent, equation (8) requires

$$
\sum \Lambda_{k}<2
$$

where

$$
\Lambda_{k} \equiv\left(\chi_{\|} / \chi_{\perp}\right)\left(b_{r, k} / B_{0 \mathrm{t}}\right)^{2}
$$

describes the radial contribution of the parallel heat conduction along the wiggling field lines relative to perpendicular transport. The fluid regime is valid for $\tau_{\perp}<\tau_{\|}<\tau_{k}$, leading to

$$
\sum \Lambda_{k}<A_{1}
$$

and

$$
\sum\left(b_{r, k} / B_{0 \mathrm{t}}\right)^{2}<A_{2},
$$

where $A_{1}=\left(k_{\perp} L_{0}\right)^{2} \sum\left(b_{r, k} / B_{0 \mathrm{t}}\right)^{2}$ and $A_{2}=\left[R q L_{q} /\left(m L_{0}^{2}\right)\right]^{2}$.

The Kadomsev-Pogutse regime is valid for $\tau_{\|}<\tau_{\perp}<\tau_{k}$, corresponding to

$$
A_{1}<\sum \Lambda_{k}<A_{3}
$$

where $A_{3}=\left[\left(\sum b_{r, k} / B_{0 \mathrm{t}}\right)\left(R q^{2} L_{q}^{2} m\right) /\left(\pi r^{3}\right)\right]^{2 / 3}$.

The Rechester-Rosenbluth regime is valid for $\tau_{k}<\tau_{\perp}$ (and $\tau_{\|}<\tau_{k}$ ), leading to

$$
\sum \Lambda_{k}>A_{3}
$$

and (16).

Assuming that the neighbouring islands have the same width, it is found from equation (9) that

$$
b_{r, k} / B_{0 \mathrm{t}}=m\left(q_{1}-q_{2}\right)^{2} \Delta^{2} /\left[16 R q^{2} q^{\prime}\right],
$$

where $q_{1}$ and $q_{2}$ are the $q$ values at the two rational surfaces. With the following parameters, $\Delta=1, R / a=3, m=3$, $L_{q}=q / q^{\prime}=a, q_{1}=3 / 2, q_{2}=4 / 3$, and $r_{s}=0.6 a$, one finds $\sum b_{r, k} / B_{0 \mathrm{t}}=6.4 \times 10^{-5}, A_{1}=5.8 \times 10^{-6}, A_{2}=2.9 \times 10^{-4}$ and $A_{3}=0.061$. By comparing (13) with (15) it is seen that, equations (8) is valid for a much higher $\chi_{\|} / \chi_{\perp}$ than equation (7) is, since $A_{1}$ is very small. The condition given by (16) is usually satisfied for tokamak plasmas. By comparing (13) with (17) and (18) it is found that the valid regime of equation (8) covers the Kadomsev-Pogutse regime and well extends into the Rechester-Rosenbluth regime.

With these different analytical results given by equations (4)-(8) as well as their different valid regimes, it is very necessary to have a comparison between them and the numerical modelling results. Basing on the earlier work of [17] for a single island and two islands (weak stochastic field), in the present paper numerical results on the heat diffusion across a local fully stochastic field are presented, to compare with the analytical result for a better understanding on the $\chi_{r}$ in the collisional regime. The effect of the perturbation amplitude and the magnetic shear on $\chi_{r}$ are also studied. 


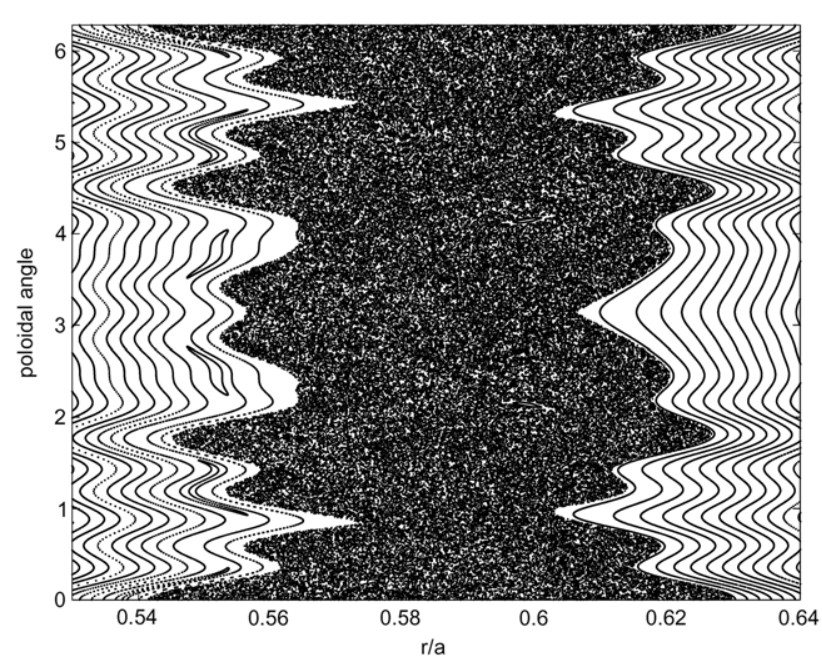

Figure 2. Local magnetic surface on the $r-\theta$ plan at $\phi=0$ for five components magnetic perturbations, $m / n=3 / 2,4 / 3,7 / 5,10 / 7$ and $11 / 8$, with $r_{3 / 2}=0.604 a, r_{4 / 3}=0.569 a, r_{7 / 4}=0.584 a$, $r_{10 / 7}=0.590 a$ and $r_{11 / 8}=0.578 a$. The perturbed field amplitude $\psi_{0}=6 \times 10^{-4}$. The local magnetic field becomes stochastic.

\section{Model and numerical modelling results}

Here the large aspect-ratio tokamak approximation is utilized. The magnetic field is defined as $\boldsymbol{B}=B_{0 \mathrm{t}} \boldsymbol{e}_{\mathrm{t}}+B_{0 \mathrm{p}}(r) \boldsymbol{e}_{\mathrm{p}}+\boldsymbol{B}_{1}$, where $B_{0 \mathrm{p}}$ is the equilibrium poloidal field, $\boldsymbol{B}_{1}=\nabla \psi \times \boldsymbol{e}_{\mathrm{t}}$ is the perturbed field, $\psi=\sum \psi_{k}(r) \cos \left(m_{k} \theta+n_{k} \phi\right)$ and $\theta$ and $\phi$ are the poloidal and toroidal angles.

The following electron energy transport equation

$\frac{3}{2} n_{\mathrm{e}} \frac{\mathrm{d} T_{\mathrm{e}}}{\mathrm{d} t}=n_{\mathrm{e}} \nabla \cdot\left(\chi_{\|} \nabla T_{\mathrm{e}}\right)+n_{\mathrm{e}} \nabla \cdot\left(\chi_{\perp} \nabla T_{\mathrm{e}}\right)+P(r)$

is solved, where $T_{\mathrm{e}}, n_{\mathrm{e}}$ and $P$ are the electron temperature, the density and the heat source, respectively. Here $n_{\mathrm{e}}, \chi_{\|}$and $\chi_{\perp}$ are assumed to be constant for simplicity, and the convective transport is neglected being valid for slowly changing islands such as tearing modes [1-4].

The 3D numerical calculation in this case is however quite challenging for high ratios of $\chi_{\|} / \chi_{\perp}$. Recently a new numerical method was developed for such a purpose, showing the required high numerical accuracy at high $\chi_{\|} / \chi_{\perp}$ [18].

Equation (20) is solved numerically with $q(r)=$ $q_{0} \exp \left(r / L_{q}\right)$ to have a constant magnetic shear along the minor radius. $\psi_{k}(r)=\psi_{0}(r / a)^{2}(1-r / a)^{2} a B_{0 \mathrm{t}}$ changing smoothly along the minor radius, which is typical for the tearing modes [1-4]. $\quad B_{0 \mathrm{t}}$ is assumed to be a constant. The heat source $P(r)=P_{0}\left[1-(r / a)^{2}\right]^{16}$ peaking at the magnetic axis. The boundary conditions are $T_{\mathrm{e}}(r=a)=T_{\mathrm{e} 0}(r=a)$ and $T_{\mathrm{e}}^{\prime}(r=0)=0$.

The local magnetic field becomes stochastic when islands of different helicity overlap. In figure 2 an example of the local magnetic surface on the $r-\theta$ plan at $\phi=0$ is shown for a case with five components magnetic perturbations, $m / n=3 / 2$, $4 / 3,7 / 5,10 / 7$ and $11 / 8$, with the rational surfaces at $r_{3 / 2}=$ $0.604 a, r_{4 / 3}=0.569 a, r_{7 / 4}=0.584 a, r_{10 / 7}=0.590 a$ and $r_{11 / 8}=0.578 a$. The perturbed field amplitude $\psi_{0}=6 \times 10^{-4}$, leading to a local stochastic magnetic field.

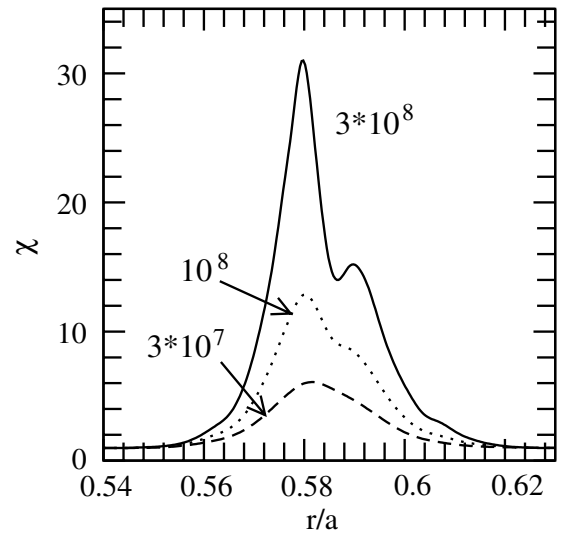

Figure 3. Corresponding to figure 2, radial profiles of $\chi=T_{\mathrm{e} 0}^{\prime} / T_{0 / 0}^{\prime}$ for $\psi_{0}=6 \times 10^{-4}$ and $\chi_{\|} / \chi_{\perp}=3 \times 10^{8}, 10^{8}$ and $3 \times 10^{7}$. A larger $\chi_{\|} / \chi_{\perp}$ leads to a larger $\chi$ in the local region.

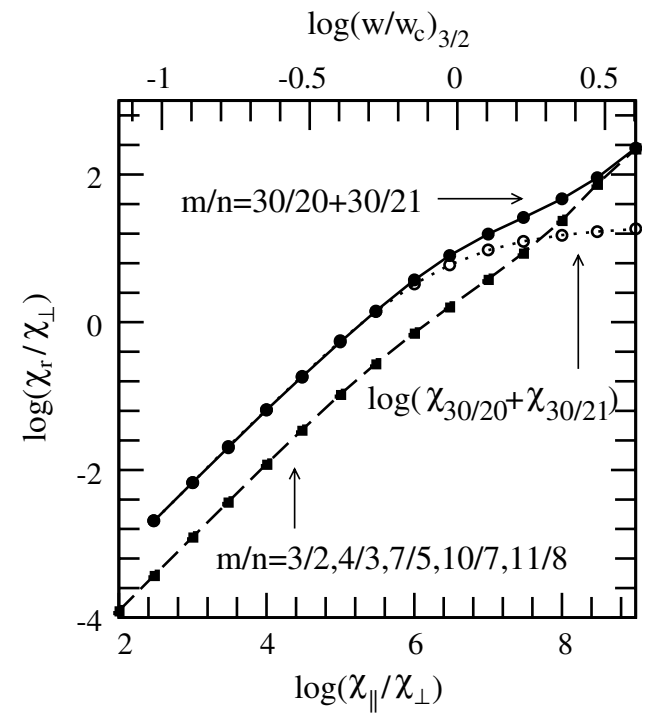

Figure 4. $\log \left(\chi_{r} / \chi_{\perp}\right)$ (solid) and $\log \left(\chi_{30 / 20}+\chi_{30 / 21}\right)$ (dotted) versus $\log \left(\chi_{\|} / \chi_{\perp}\right)$ for $\psi_{0}=9 \times 10^{-4}$ and $m / n=30 / 20$ and $30 / 21$. The value of $\chi$ is taken at the middle between these two rational surfaces. The dashed curve is $\left.\log \left(\chi_{r} / \chi_{\perp}\right)\right|_{r=0.579 a}$ for the five island case with $m / n=3 / 2,4 / 3,7 / 5,10 / 7$, and $11 / 8$. The upper horizontal axis shows the $\log \left(W / W_{c}\right)_{m / n=3 / 2}$.

Defining an normalized effective radial heat conductivity to be

$$
\chi \equiv \chi_{\mathrm{e}} / \chi_{\perp}=\chi_{r}+1,
$$

corresponding to figure 2 , in figure 3 radial profiles of $\chi$ are shown for $\chi_{\|} / \chi_{\perp}=3 \times 10^{8}, 10^{8}$ and $3 \times 10^{7}$. As expected, a larger $\chi_{\|} / \chi_{\perp}$ leads to a larger $\chi$ in the local stochastic field region, and $\chi=1$ ((or $\chi_{r} \sim 0$ ) away from this region. $\chi_{\perp}$ is kept constant in all our calculations.

In figure $4 \log \left(\chi_{r} / \chi_{\perp}\right)$ versus $\log \left(\chi_{\|} / \chi_{\perp}\right)$ is shown for $L_{q}=0.3 a$ and $\psi_{0}=9 \times 10^{-4}$ by the solid curve (with solid circles) for a two island case, $m / n=30 / 20$ and 30/21, leading to the island width of $W_{30 / 20}=W_{30 / 21}=0.045 a$. The rational surfaces are at $r_{30 / 20}=0.604 a$ and $r_{30 / 21}=0.589 a$, and the parameter $\Delta$ characterizing the ergodicity is 3.0. The value of $\chi$ is taken at the middle between these two rational surfaces. The upper horizontal axis shows the corresponding values of 


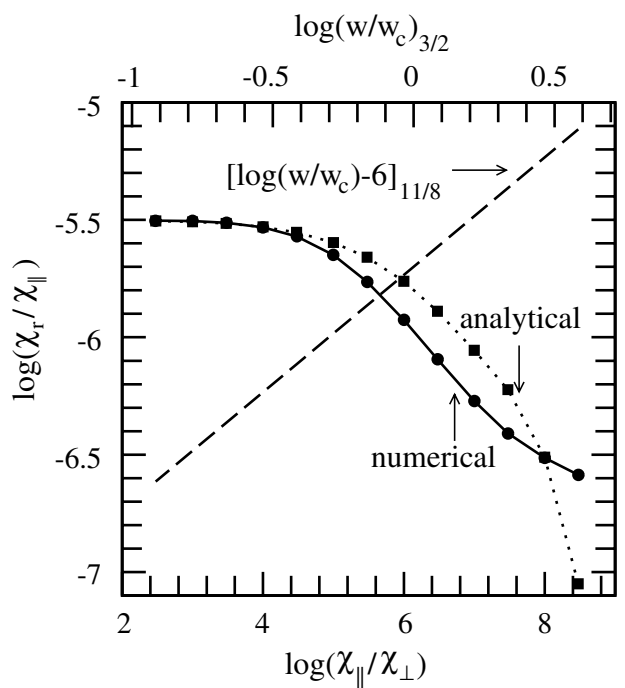

Figure 5. Numerical results (solid) and the analytical results of equation (8) (dotted) of $\log \left(\chi_{r} / \chi_{\|}\right)$at $r=0.595 a$ versus $\log \left(\chi_{\|} / \chi_{\perp}\right)$ for the five island case, with $L_{q}=0.3 a$ and $\psi_{0}=1.5 \times 10^{-3}$. The dashed curve shows $\left[\log \left(W / W_{c}\right)-6\right]_{m / n=11 / 8}$. The upper horizontal axis is the $\log \left(W / W_{c}\right)_{m / n=3 / 2}$.

the $\log \left(W / W_{c}\right)$ for the $m / n=3 / 2$ component, being equal to that of the $m / n=30 / 20$ component. The dotted curve (with empty circles) shows $\log \left(\chi_{30 / 20}+\chi_{30 / 21}\right)$, where $\chi_{30 / 20}\left(\chi_{30 / 21}\right)$ is the $\left(\chi_{r} / \chi_{\perp}\right)$ obtained for a single $30 / 20(30 / 21)$ island alone at the same radial location, with other parameters unchanged. For $\chi_{\|} / \chi_{\perp}<10^{6}\left(W<0.7 W_{c}\right), \chi_{r} \propto \chi_{\|}$and the dotted curve is the same as the solid one, as predicted by equation (8) that $\chi_{r}$ is determined by the additive effects of the individual islands for $W_{k}<W_{c, k}$. For $\chi_{\|} / \chi_{\perp}>3 \times 10^{8}\left(W>3 W_{c}\right), \chi_{r}$ also approximately scales as $\chi_{\|}$. Between these two limits there is a transition region where $\chi_{r}$ slowly increases with $\chi_{\|}$. The dashed curve with squares in figure 4 is the $\log \left(\chi_{r} / \chi_{\perp}\right)$ at $r=0.579 a$ for the five components magnetic perturbations, $m / n=3 / 2,4 / 3,7 / 5,10 / 7$ and $11 / 8$, with $\psi_{0}=9 \times 10^{-4}$ and $\Delta$ ranging from 1.6 to 3.8 . It shows a similar behaviour as the two island cases, $\chi_{r}$ scales as $\chi_{\|}$for a low or high ratio of $\chi_{\|} / \chi_{\perp}$, and between these two limits there is a transition region around $W_{k} \sim W_{c, k}$ where $\chi_{r}$ slowly increases with $\chi_{\|}$.

The local value of $\chi_{r}$ at $r=0.595 a$ obtained from numerical calculations (solid curve) is compared with the analytical results from equation (8) (dotted curve) for the five island case in figure 5 , where $\log \left(\chi_{r} / \chi_{\|}\right)$is shown as a function of $\log \left(\chi_{\|} / \chi_{\perp}\right)$ for $L_{q}=0.3 a$ and $\psi_{0}=1.5 \times 10^{-3}$. The upper horizontal axis shows the corresponding values of the $\log \left(W / W_{c}\right)$ for the $m / n=3 / 2$ component. The dashed curve shows $\left[\log \left(W / W_{c}\right)-6\right]$ for the $m / n=11 / 8$ component, being a little different from that of the $m / n=3 / 2$ component due to different mode numbers. The numerical results agree with the analytical ones for $W<W_{c}$. With increasing $\chi_{\|} / \chi_{\perp}$ (or $W / W_{c}$ ), both the analytical and the numerical results decrease. The decrease in the analytical $\chi_{r}$ is due to the decrease in $W_{c}$ for larger $\chi_{\|} / \chi_{\perp}$, leading to a larger $z_{k}$ and a corresponding smaller $g_{k}$, as seen from figure 1 . The numerical results decrease by about one order of magnitude from the region $W \ll W_{c}$ to the region $W \approx 3 W_{c}$.

The above results shown in figures 4 and 5 are only for the $\chi_{r}$ at a few radial locations. Since $\chi_{r}$ is not constant across the

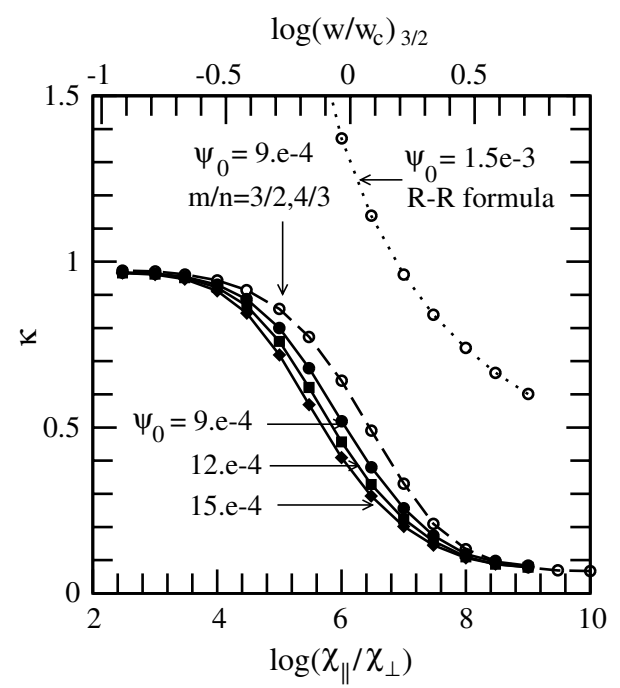

Figure 6. $\kappa \equiv\left\langle\chi_{r}\right\rangle /\left[0.5 \chi_{\|} \sum\left(b_{r, k} / B_{0 \mathrm{t}}\right)^{2}\right]$ versus $\log \left(\chi_{\|} / \chi_{\perp}\right)$ for $\psi_{0}=9 \times 10^{-4}, 1.2 \times 10^{-3}$ and $1.5 \times 10^{-3}$ (solid), $\kappa$ calculated from equation (4) for $\psi_{0}=1.5 \times 10^{-3}$ (dotted) and $\kappa$ for $\psi_{0}=9 \times 10^{-4}$ and $m / n=3 / 2$ and $4 / 3$ (dashed). The upper horizontal axis is the $\log \left(W / W_{c}\right)_{m / n=3 / 2}$ for $\psi_{0}=1.5 \times 10^{-3}$.

stochastic field region as seen from figure 3 , in the following the radial averaged $\chi_{r},\left\langle\chi_{r}\right\rangle=\int \chi_{r} \mathrm{~d} r /\left(r_{b}-r_{a}\right)$, is used for a further comparison with analytical results. The integration is taken from $r_{a}=0.575 a$ to $r_{b}=0.600 a$ where the magnetic field is fully stochastic. Defining

$$
\kappa \equiv\left\langle\chi_{r}\right\rangle /\left[0.5 \chi_{\|} \sum\left(b_{r, k} / B_{0 \mathrm{t}}\right)^{2}\right],
$$

being the average radial enhancement relative to the $\chi_{r}$ given by equation (8) by neglecting the function $g_{k}\left(z_{k}\right)$, in figure $6 \kappa$ is shown as a function of $\log \left(\chi_{\|} / \chi_{\perp}\right)$ by the solid curves with $\psi_{0}=9 \times 10^{-4}, 1.2 \times 10^{-3}$ and $1.5 \times 10^{-3}$ for the five island case: $m / n=3 / 2,4 / 3,7 / 5,10 / 7$ and $11 / 8$. The upper horizontal axis shows the corresponding values of the $\log \left(W / W_{c}\right)$ for the $m / n=3 / 2$ component with $\psi_{0}=1.5 \times 10^{-3}$. For $\chi_{\|} / \chi_{\perp} \sim 10^{2}-10^{3}\left(W_{k} / W_{c, k} \ll 1\right)$, $\kappa=1$ for different $\psi_{0}$, as predicted by equation (8) in the limit $z_{k}=0$. With the increase in $\chi_{\|} / \chi_{\perp}, \kappa$ decreases and approaches a steady value again at high $\chi_{\|} / \chi_{\perp}\left(W_{k} / W_{c, k} \sim 5\right)$ for sufficiently large $\psi_{0}$ ( $\kappa$ oscillates for small $\left.\psi_{0}\right)$. This differs from the prediction of equation (5) that $\kappa \sim 1 / L_{k} \sim$ $D_{\mathrm{M}}^{1 / 3} \sim \psi_{0}^{2 / 3}$. The faster decay of $\kappa$ with increasing $\chi_{\|} / \chi_{\perp}$ for a larger $\psi_{0}$ is due to the corresponding larger $W_{k} / W_{c, k}$ so that the transition region as shown in figure 4 is reached at a lower $\chi_{\|} / \chi_{\perp}$. The dotted curve in figure 6 shows the analytical result of Rechester-Rosenbluth from equation (4) with $\psi_{0}=1.5 \times 10^{-3}$, being different from the numerical results. The dashed curve is the $\kappa$ for the two island case, $m / n=3 / 2$ and $4 / 3$, with $\psi_{0}=9.0 \times 10^{-4}$ and the radial average from $r=0.58 a$ to $0.59 \mathrm{a}$, which shows a similar behaviour as the five island case: $\kappa$ decreases by about one order of magnitude from small to large $W_{k} / W_{c, k}$.

Increasing the magnetic shear by 3 times to $L_{q}=0.1 a$, $\kappa$ versus $\log \left(\chi_{\|} / \chi_{\perp}\right)$ is shown in figure 7 by the solid curves for the five island case with $\psi_{0}=1.5 \times 10^{-3}, 4.5 \times 10^{-3}$ and $7.5 \times 10^{-3}$. The upper horizontal axis shows the corresponding values of the $\log \left(W / W_{c}\right)$ for the $m / n=3 / 2$ component with 


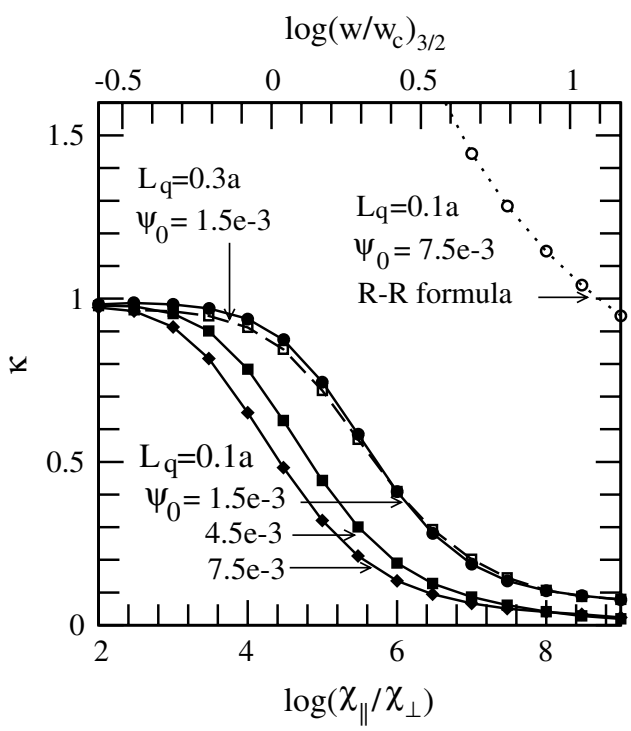

Figure 7. $\kappa$ versus $\log \left(\chi_{\|} / \chi_{\perp}\right)$ for $L_{q}=0.1 a$ and $\psi_{0}=1.5 \times 10^{-3}$, $4.5 \times 10^{-3}$ and $7.5 \times 10^{-3}$ (solid), $\kappa$ from equation (4) for $\psi_{0}=7.5 \times 10^{-3}$ (dotted) and $\kappa$ for $L_{q}=0.3 a$ and $\psi_{0}=1.5 \times 10^{-3}$ (dashed). The upper horizontal axis shows the $\log \left(W / W_{c}\right)_{m / n=3 / 2}$ with $\psi_{0}=4.5 \times 10^{-3}$ and $L_{q}=0.1 a$.

$\psi_{0}=4.5 \times 10^{-3}$. In this case the rational surfaces are closer, with $r_{3 / 2}=0.595 a, r_{4 / 3}=0.583 a, r_{7 / 4}=0.588 a$, $r_{10 / 7}=0.590 a$ and $r_{11 / 8}=0.587 a$, and the radial average is from $0.584 a$ to $0.594 a$. It is seen that $\kappa$ approaches 1 for $\chi_{\|} / \chi_{\perp} \sim 10^{2}\left(W \ll W_{c}\right)$ and a nearly steady value at large $\chi_{\|} / \chi_{\perp}\left(W=10 W_{c}\right)$ for sufficiently large $\psi_{0}$, similarly to figure 6 . The dotted curve is the result from equation (4) for $\psi_{0}=7.5 \times 10^{-3}$, being more different from the numerical results for the large magnetic shear case. The dashed curve is the result with a smaller magnetic shear, $L_{q}=0.3 a$, and $\psi_{0}=1.5 \times 10^{-3}$. When comparing figures 6 and 7 it is seen that with a large magnetic shear $\kappa$ converges to a smaller value at large $\chi_{\|} / \chi_{\perp}$ for sufficiently large $\psi_{0}$, differing from the prediction of equation (5), $\kappa \sim 1 / L_{k} \sim L_{q}^{-2 / 3}$.

\section{Discussion and summary}

Using a numerical approach the heat diffusion across a local fully stochastic field is studied in the collisional regime. Comparing the numerical results with the analytical ones given by equations (4)-(8), it is seen that the parameter $W_{k} / W_{c, k}$ not considered in $[10,11]$ is important in characterizing the transport even for a fully stochastic field. For $\mathrm{Wk} / \mathrm{Wc}, \mathrm{k}<1$ the analytical result given by equation (8) is found to agree with numerical results, while for $W_{k} \gg W_{c, k}, \chi_{r}$ approximately scales with $\chi_{\|}$. These results extends the earlier work for a weak stochastic field [17].

The fluid regime result is shown to be incorrect not only in its form of $\chi_{r}$ but also in its valid regime. The KadomsevPogutse regime is not found from numerical results. In fact, the scaling $\chi_{r} \sim\left(\chi_{\|} / \chi_{\perp}\right)^{1 / 2}$ given by equation (6) is seen from the numerical calculations only at the rational surface for a single island satisfying $W \gg W_{c}$ [17]. As for the Rechester-Rosenbluth regime, the scaling $\chi_{r} \sim \chi_{\|}$is found for a sufficiently high $\chi_{\|} / \chi_{\perp}$ from the numerical results, but the scaling with the magnetic shear and the perturbation amplitude is different. Recent numerical studies have shown that the magnetic field shear plays an important role in the spacial diffusion of the field lines, and the excursions of field lines significantly differ from Brownian motions [14]. On the other hand it should be noted that in our model the local stochastic field results from the overlap of several islands, while in $[10,11]$ an infinite fully stochastic field is assumed. Future calculations in a non-local stochastic field will be helpful for a further comparison with the Rechester-Rosenbluth regime.

Equation (8) has an important implication in heat diffusion across a stochastic field where $\chi_{\|} / \chi_{\perp}$ is not large enough. For typical tokamak edge parameters $T_{\mathrm{e}}=40 \mathrm{eV}, n_{\mathrm{e}}=10^{19} \mathrm{~m}^{-3}$, $L_{q}=a, R / a=3, n=2$ and $\chi_{\perp}=1 \mathrm{~m}^{2} \mathrm{~s}^{-1}$ (assuming the perpendicular heat transport to be anomalous), one finds $W_{c}=0.060 a$ by using the classical parallel electron heat conductivity $\chi_{\| c}=3.16 v_{\mathrm{Te}} \lambda_{\mathrm{e}}$. This means that for smaller islands $(W<0.060 a)$ the field ergodicity plays no role, and the heat diffusion is determined by the additive effect of individual islands. Only for sufficiently large islands or higher plasma temperature the field ergodicity dominates the radial transport.

Here $\chi_{\|}$is taken to be a constant being valid for the collisional regime. For the collisionless regime $\chi_{\|}=\chi_{\| c}[1+$ $\left.\left(3.16 \lambda_{\mathrm{e}} k_{\|}\right)^{2}\right]^{-1 / 2}$ could be used $[19,20]$. Such a $\chi_{\|}$reduces to $\chi_{\| c}$ for $\lambda_{\mathrm{e}} k_{\|} \ll 1$ and to $v_{\mathrm{Te}} / k_{\|}$in the opposite limit. The heat transport is studied here with given perturbed magnetic fields. It was found recently that drift-tearing modes with high mode numbers can be driven unstable by the electron temperature gradient [5]. Further investigation on the nonlinear mode saturation is necessary for calculating their effect on the transport.

In summary, the heat diffusion across a local fully stochastic magnetic field is studied numerically. It is found that the numerical results agree with the analytical ones given by equation (8) in the quasi-linear regime $W_{k}<W_{c, k}$. For $W_{k} \gg W_{c, k}$, the normalized (to $\chi_{\|}$and the square of the radial magnetic perturbation) enhanced heat conductivity $\kappa$ decreases by about one order of magnitude and is smaller for a larger magnetic shear, and $\kappa$ is independent of $\chi_{\|}$and the magnetic perturbation amplitude if the perturbation amplitude is sufficiently large, providing a simple way for estimating the magnitude of the enhanced radial heat conductivity in a local fully stochastic field.

\section{Reference}

[1] Wesson J.A. et al 1989 Nucl. Fusion 29641

[2] Chang Z. et al 1995 Phys. Rev. Lett. 744663

[3] Günter S. et al 2001 Phys. Rev. Lett. 87275001

[4] Yu Q. et al 2000 Nucl. Fusion 402031

[5] Yu Q., Günter S. and Scott B.D. 2003 Phys. Plasmas 10797

[6] Wolf R.C. et al 2005 Nucl. Fusion 451700

[7] Evans T.E. et al 2004 Phys. Rev. Lett. 92235003

[8] Strumberger E. 1999 J. Nucl. Mater. 266-269 1207

[9] Ghendrih Ph., Grosman A. and Capes H. 1996 Plasma Phys. Control. Fusion 381653

[10] Rechester A.B. and Rosenbluth M.N. 1978 Phys. Rev. Lett. 4038

[11] Krommers J.A., Oberman C. and Kleva R.G. 1983 J. Plasma Phys. 3011

[12] Stix T.H. 1978 Nucl. Fusion 18353

[13] Kadomtsev B.B. and Pogutse O.P. 1979 Proc. 7th IAEA Conf. on Plasma Physics and Controlled Fusion Research (Innsbruck, 1978) (Vienna: IAEA) vol I, p 649 
[14] de Rover M., Schilham A.M.R., Montvai A. and Lopes Cardozo N.J. 1999 Phys. Plasmas 62443

[15] Bickerton R.J. 1997 Plasma Phys. Control. Fusion 39339

[16] Liewer P.C. 1985 Nucl. Fusion 25543

[17] Yu Q. 2006 Phys. Plasmas 13062310
[18] Günter S., Yu Q., Krüger J. and Lackner K. 2005 J. Comput. Phys. 209354

19] Yu Q., Günter S., Giruzzi G., Lackner K. and Zabiego M. 2000 Phys. Plasmas 7312

[20] Chang Z. and Callen J.D. 1992 Phys. Fluids B 41167 